\title{
Regulatory Approvals of RET Inhibitors for NSCLC and Thyroid Cancer ESMO Updates the Guidelines for the Diagnosis of RET-Altered Cancer to Herald a New Era in Targeted Therapy
}

\author{
Alfredo Addeo ${ }^{*}$, Frank Stenner²
}

The RET gene encodes a tyrosine kinase receptor that regulates cell survival and proliferation by triggering key downstream pathways. ${ }^{1}$ RET gene mutations and RET gene fusions are involved in the pathogenesis of lung, thyroid and other cancers. Recently, in 2021, the European Society for Medical Oncology (ESMO) has developed recommendations for the laboratory diagnosis of targetable RET rearrangements and mutations. ${ }^{2}$ Following the results from phase I/II clinical trials ${ }^{3,4}$, selpercatinib, a RET tyrosine kinase inhibitor (TKI), received the FDA $^{5}$ and Swissmedic ${ }^{6}$ authorization for the treatment of adult patients with RET fusionpositive non-small cell lung cancer (NSCLC) and advanced RET fusion-positive thyroid cancer who require systemic therapy and who have progressed after prior treatment. Selpercatinib is also indicated for the treatment of adults and adolescents $\geq 12$ years old with advanced RET-mutated medullary thyroid carcinoma (MTC) who require systemic therapy and who have experienced progression after prior treatment with TKIs. In this clinical setting, the Food and Drug Administration (FDA) has recently approved another RET TKI, pralsetinib. ${ }^{7}$ In addition, the FDA also granted accelerated approval to pralsetinib for adult patients with metastatic RET fusion-positive NSCLC. ${ }^{8}$ This review aims to summarize the new ESMO recommendations for the laboratory diagnosis of RET-altered cancers and the clinical trial data that support the regulatory approvals of selpercatinib and pralsetinib for RET-altered NSCLC and thyroid cancer.

\section{Keywords: RET inhibitors, selpercatinib, pralsetinib, NSCLC, thyroid cancer}

RET-altered cancers with RET gene mutations and gene fusions

The proto-oncogene rearranged during transfection (RET) gene is located on the long arm of chromosome 10 and encodes a transmembrane glycoprotein receptor with tyrosine kinase activity. ${ }^{1}$ Activation of the RET receptor occurs via autophosphorylation of intracellular tyrosine residues. RET receptor activation triggers several downstream pathways that include PI3K-AKT, RAS-MAPK, JAK-STAT, PKC, and PLC $\gamma$, which have key roles
${ }^{1}$ Oncology Department, University Hospital Geneva, Geneva, Switzerland 2 Department of Medical Oncology, University Hospital Basel, Basel,

Switzerland

${ }^{*}$ Corresponding Author:

Dr Alfredo Addeo

Oncology Department, University

Hospital Geneva

Geneva, Switzerland

Email: alfredo.addeo@hcuge.ch

Tel: +41(0)223729862

DOI: $10.36000 / \mathrm{hbT} . O H .2021 .08 .037$ ISSN: 2673-2092 (Print) and 26732106 (Online)

This article was received on May 26, 2021

This article was accepted on June 07, 2021

This article was published on June 11, 2021

Addeo A, Stenner F. ESMO Updates the Guidelines for the Diagnosis of RET-Altered Cancer to Herald a New Era in Targeted Therapy. healthbook TIMES Onco Hema. 2021; (8): 68-73 in cell survival and proliferation (Figure 1). RET gene alterations have been identified in many solid tumors and include RET gene mutations and RET gene fusions, termed RET-altered cancers. ${ }^{1,2}$ RET point mutations are associated with medullary thyroid cancer (MTC), whereas RET fusions are found in 10\% of papillary thyroid carcinoma and $1-2 \%$ of lung adenocarcinoma (Figure 2). ${ }^{9}$ 
RET Inhibitors for NSCLC and Thyroid Cancer

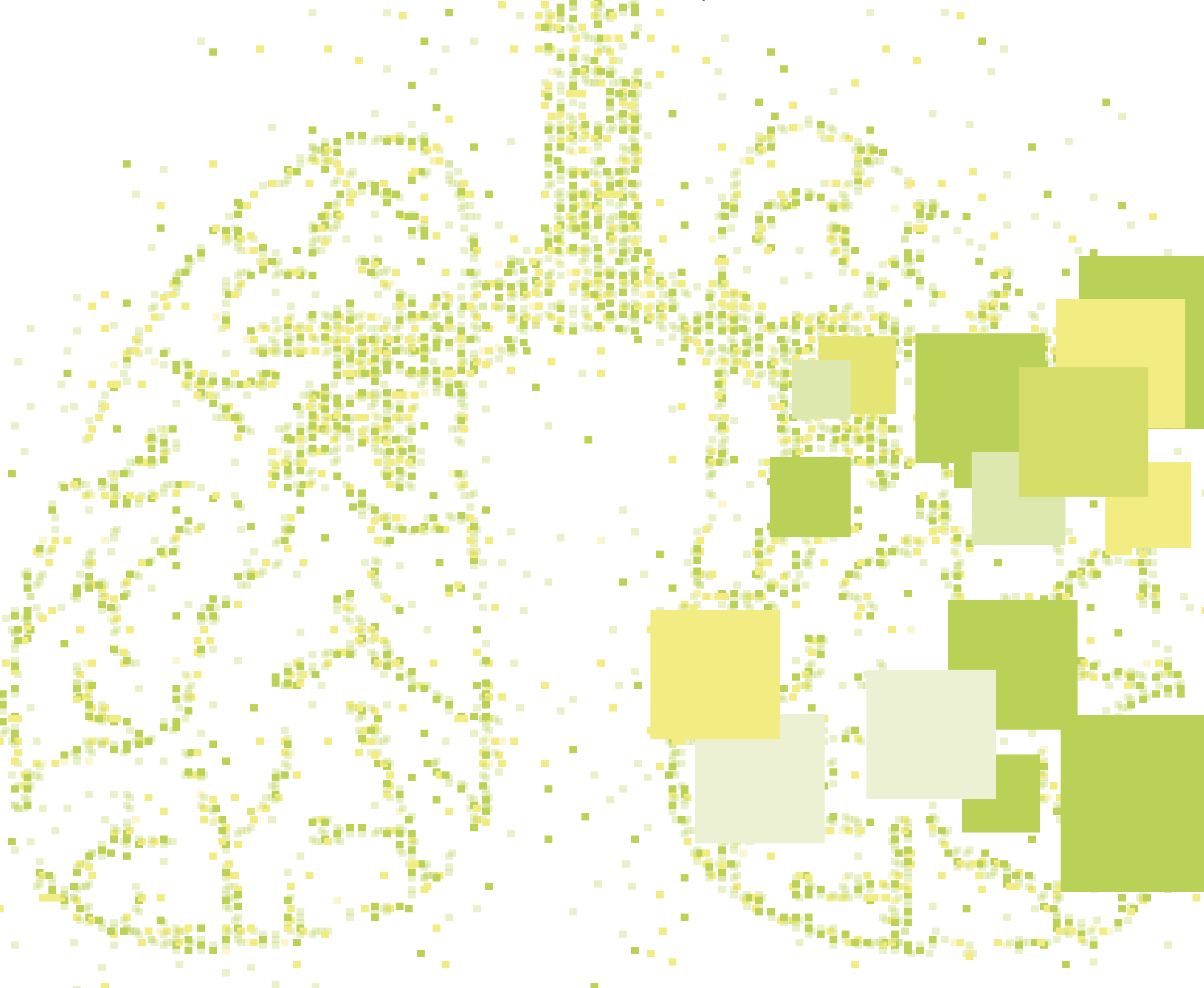

Table 1. The European Society for Medical Oncology (ESMO) Translational Research and Precision Medicine Working Group recent recommendations for currently available techniques for routine clinical laboratory detection of targetable RET rearrangements and mutations, with their strengths and limitations. ${ }^{a}$ False-positive up to $40 \%$. ${ }^{b}$ False-negative up to $40 \%$. 'Requires the use of specific fusion partner probe. ${ }^{\text {dU }}$ Use in settings with several possible fusion partners,

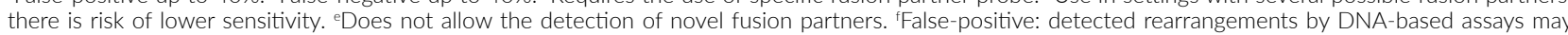
not result in fusions, and correlation with RNA-based confirmation of predicted fusion transcript is required. BFalse-negative: some introns involved in RET rearrangements may be inadequately evaluated, for technical reasons. hindication on the in-frame nature of the fusion (functionality). DNA-seq NGS, DNA sequencing by next-generation sequencing; FISH, fluorescent in situ hybridization; IHC, immunohistochemistry; RNA-seq NGS, RNA sequencing by nextgeneration sequencing; RT-PCR, reverse transcription-polymerase chain reaction. Adapted from Belli et al. 2021. ${ }^{2}$

\begin{tabular}{|c|c|c|c|c|c|}
\hline Method & Sensitivity & Specificity & $\begin{array}{c}\text { Detection of } \\
\text { partner }\end{array}$ & $\begin{array}{c}\text { Detection of } \\
\text { expression }\end{array}$ & $\begin{array}{l}\text { Use in } \\
\text { screening }\end{array}$ \\
\hline IHC & moderate ${ }^{a}$ & moderate $^{b}$ & no & yes & no \\
\hline FISH & high & high & no/yes ${ }^{c}$ & no & rarely \\
\hline RT-PCR & moderate/highd & high & yes/noe & yes & rarely \\
\hline DNA-seq NGS & moderatef & high/moderateg & yes & no & yes \\
\hline RNA-seq NGS & high & high & yes & yes $^{h}$ & yes \\
\hline
\end{tabular}




\section{MINI REVIEW}

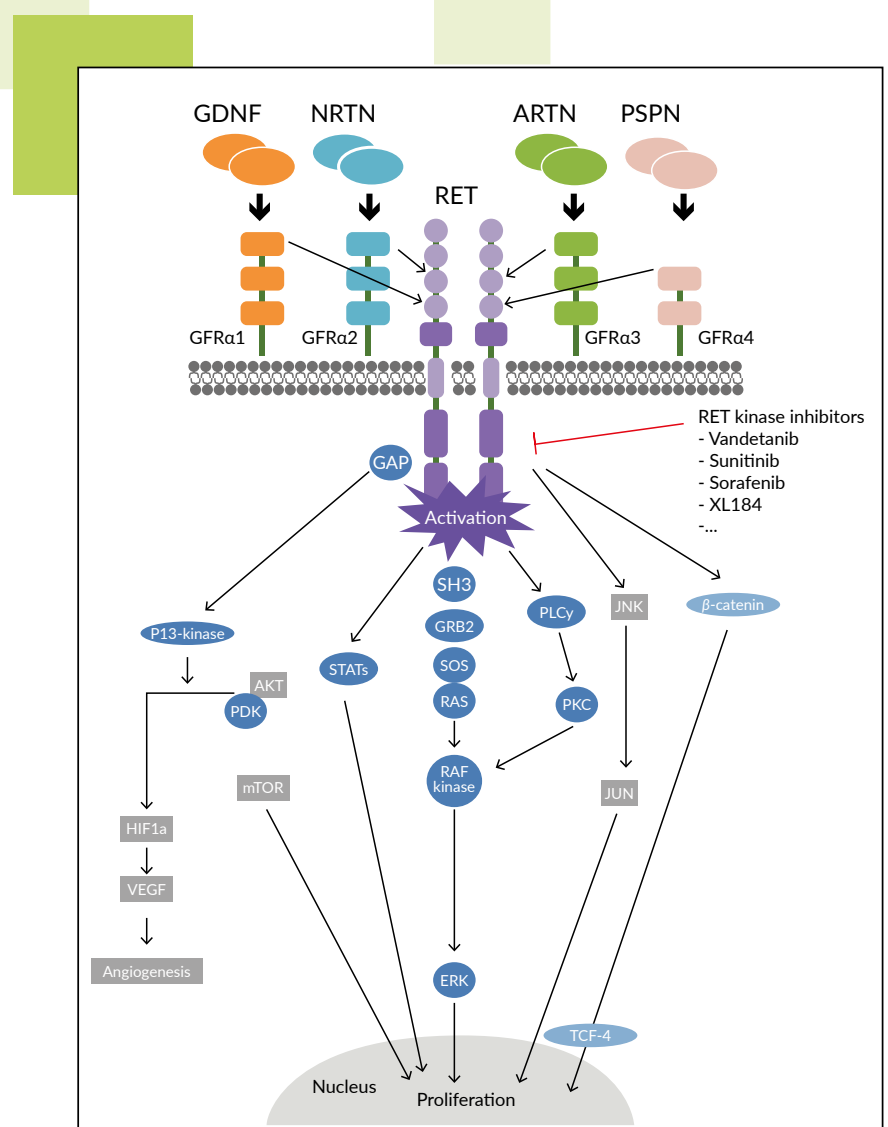

Figure 1. Outline of RET signaling pathways. Adapted from Prazeres et al. 2011. ${ }^{21}$

The most common somatic mutation in sporadic MTC is M918T, which is present in up to $40 \%$ of MTC patients and is associated with disease aggressiveness. ${ }^{10}$ Other single amino acid changes might occur at residues C611, C618, C620, C630, C634, E768, A883 and S891.

\section{The 2021 ESMO guidelines for the diagnosis of RET-altered} cancer

Accurate detection of RET gene fusions and mutations is the basis for targeted therapy with the new RET inhibitors and essentials to ensure personalized medicine. Similar to regulatory approval for drug treatment, diagnostics require regulatory evaluation and authorization. The ESMO Translational Research and Precision Medicine Working Group has recently issued recommendations for the routine clinical detection of targetable RET rearrangements and mutations (Table 1). ${ }^{2}$ The main methods were immunohistochemistry (IHC), fluorescent in situ hybridization (FISH), reverse transcription-polymerase chain reaction (RT$P C R$ ), DNA sequencing by next-generation sequencing (DNAseq NGS), and RNA sequencing by next-generation sequencing (RNA-seq NGS). The ESMO guidelines highlight that $\mathrm{IHC}$ alone is not suitable as a routine diagnostic screening tool to identify RET alterations due to lack of sensitivity and specificity and lack of standardization of laboratory methods. Recently, in 2021, ESMO has developed recommendations for RET testing of malignant tumors to identify patients eligible for anti-RET therapy (Table 1).
The rationale for selective RET inhibitors as targeted therapy in NSCLC and thyroid carcinoma

An early approach to targeting therapy in RET-altered solid cancer was made with multikinase inhibitors (MKIs) with anti-RET activities. ${ }^{2}$ However, the limitations of using MKIs include low target specificity and high frequency of side effects due to offtarget toxicities. In 2012, vandetanib, a small-molecule inhibitor of vascular endothelial growth factor receptor (VEGFR), epidermal growth factor receptor (EGFR) and RET tyrosine kinases, was approved by Swissmedic for the treatment of patients with unresectable, rapidly progressive and symptomatic MTC. ${ }^{11}$ This approval was based on the results from a phase III study, which demonstrated that vandetanib versus placebo was associated with significantly prolonged PFS and improved response rates in patients with MTC. ${ }^{12}$ Unlike selective RET inhibitors however, vandetanib only shows moderate efficacy in the treatment of NSCLC harboring RET rearrangement. ${ }^{13}$

New highly selective RET inhibitors have recently been approved based on evidence of safety and efficacy from randomized clinical trials (RCTs). ${ }^{3-5,8,14}$ The importance of accurate and widely available diagnostics has been highlighted by the regulatory approvals of selpercatinib (Swissmedic and FDA) and pralsetinib (FDA only), two selective RET inhibitors, following the results of phase I/II RCTs.

In Switzerland, despite federal authorization of selpercatinib for the treatment of RET fusion-positive NSCLC, reimbursement has to be requested at the insurance company through article $71 \mathrm{~b}$ of the Health Insurance Ordinance (KVV/OAMal). For pralsetinib, which is currently not approved in Switzerland for treatment of RET fusion-positive NSCLC, reimbursement can be requested through article 71c. For patients with thyroid cancer, vandetanib is an alternative therapy reimbursed though insurance.

\section{Selpercatinib for the treatment of RET fusion-positive NSCLC and thyroid cancer, and RET mutation-positive MTC}

Selpercatinib (LOXO-292) is an orally bioavailable, selective inhibitor of wildtype, mutant, and fusion products of RET. ${ }^{5}$ Regulatory approval of selpercatinib was based on the results from the open-label, phase I/II LIBRETTO-001 trial in patients with advanced solid tumors, RET fusion-positive solid tumors, and MTC. $3,4,6,15$

LIBRETTO-001 included 105 patients with RET fusion-positive NSCLC who were pretreated with platinum chemotherapy. ${ }^{3}$ All study participants had confirmed RET fusion status using either RT-PCR, NGS, or FISH. In phase I portion of the study, patients were treated with oral selpercatinib at $20 \mathrm{mg}$ once daily to 240 mg twice daily $(n=49)$. In the phase II study, patients received $160 \mathrm{mg}$ of selpercatinib twice daily $(\mathrm{n}=56)$. The results showed an overall response rate (ORR) of $64 \%$ (95\% Cl: 54-73\%), regardless of previous $\mathrm{MKI}$ or immune checkpoint inhibitor (ICI) therapy (Figure 3). The median duration of response (DoR) was 


\section{RET Inhibitors for NSCLC and Thyroid Cancer}

17.5 months (95\% Cl: 12 months-not estimable [NE]). In 39 treatment-naive patients, the ORR was 85\% (95\% Cl: 70-94\%), and the median DoR was not achieved. This trial included 11 pretreated RET fusion-positive patients with cerebral metastases diagnosed at study entry. ${ }^{8}$ A disease response occurred in $91 \%$ (10/11) of patients, including 3 complete responses (27\%). These data provide evidence that selpercatinib could cross the bloodbrain barrier to reach the central nervous system (CNS).

Somatic RET mutations occur in more than $65 \%$ of sporadic MTCs and up to $90 \%$ of advanced MTC. ${ }^{16}$ The LIBRETTO-001 trial included 55 patients with RET mutation-positive MTC who were previously treated with vandetanib, cabozantinib, or both. ${ }^{4}$ The objective response rate (ORR) was 69\% (95\% Cl: 55-81\%), and the 1-year progression-free survival (PFS) was $82 \%$ (95\% Cl: 69-90\%). The study also included 88 treatment-naive patients with RET-mutant MTC. Among them, the ORR was $73 \%(95 \% \mathrm{Cl}$ : $62-82 \%)$, and the 1-year PFS was 92\% (95\% Cl: 82-97\%). There were also 19 patients with previously treated RET fusion-positive thyroid cancer, with a response rate of 79\% (95\% Cl: 54-94\%) and a 1-year PFS rate of $64 \%$ (95\% Cl: 37-82\%). The most common adverse events (AEs) of grade 3 or more were hypertension (21\%), increased serum levels of alanine aminotransferase (ALT) (11\%), increased serum aspartate aminotransferase (AST) (9\%), hyponatremia (8\%), and diarrhea (6\%).

In a post hoc analysis of LIBRETTO-001, selpercatinib demonstrated a favorable safety profile, with low-grade treatment-emergent adverse effects (TEAEs) and early-onset toxicities that were clinically manageable, resulting in low rates of discontinuation of treatment. ${ }^{17}$ In this study, the safety population consisted of 702 patients who received at least one dose of selpercatinib, with a follow-up until the cutoff date. The majority of TEAEs were low grade and included dry mouth (38.9\%), diarrhea (36.6\%) and hypertension (35.9\%).Grade 3-4 TEAEs occurred in $59.1 \%$ of patients and included hypertension (17.1\%), increased serum levels of AST (7.4\%) and ALT (9.1\%), and QTC prolongation (4.0\%), which were manageable with dose adjustments. TEAEs that resulted in dose reduction or drug interruption were dry mouth, diarrhea and hypertension.

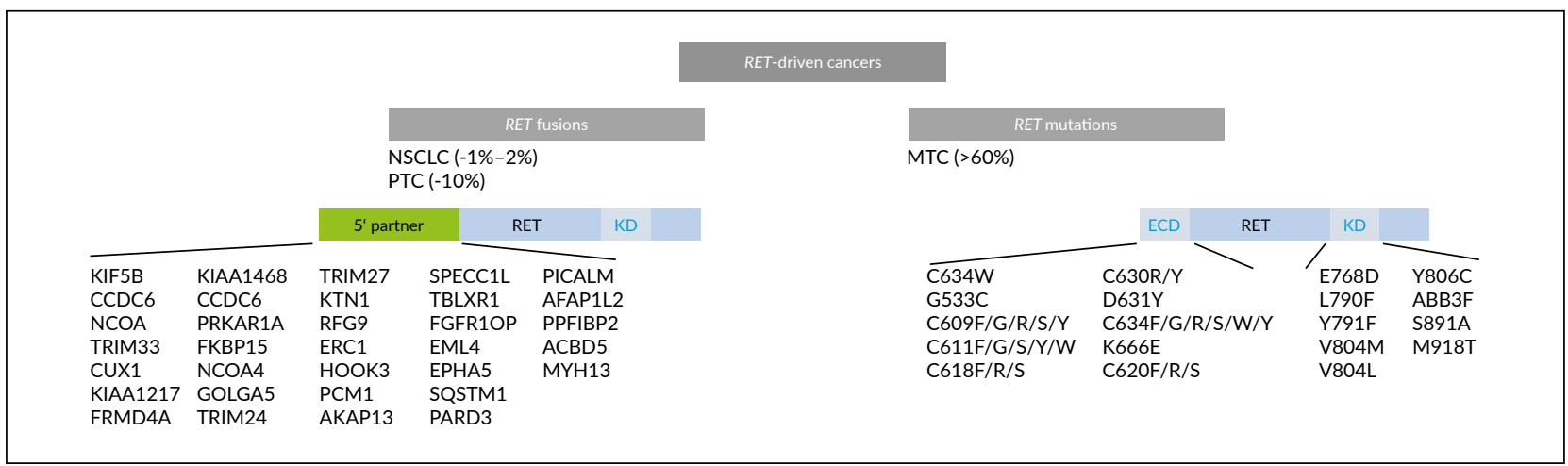

Figure 2. RET alterations by mutation type in non-small cell lung cancer (NSCLC), papillary thyroid cancer (PTC) and medullary thyroid cancer (MTC). ECD, extracellular domain; KD, kinase domain. Adapted from lams et al. 2018.9

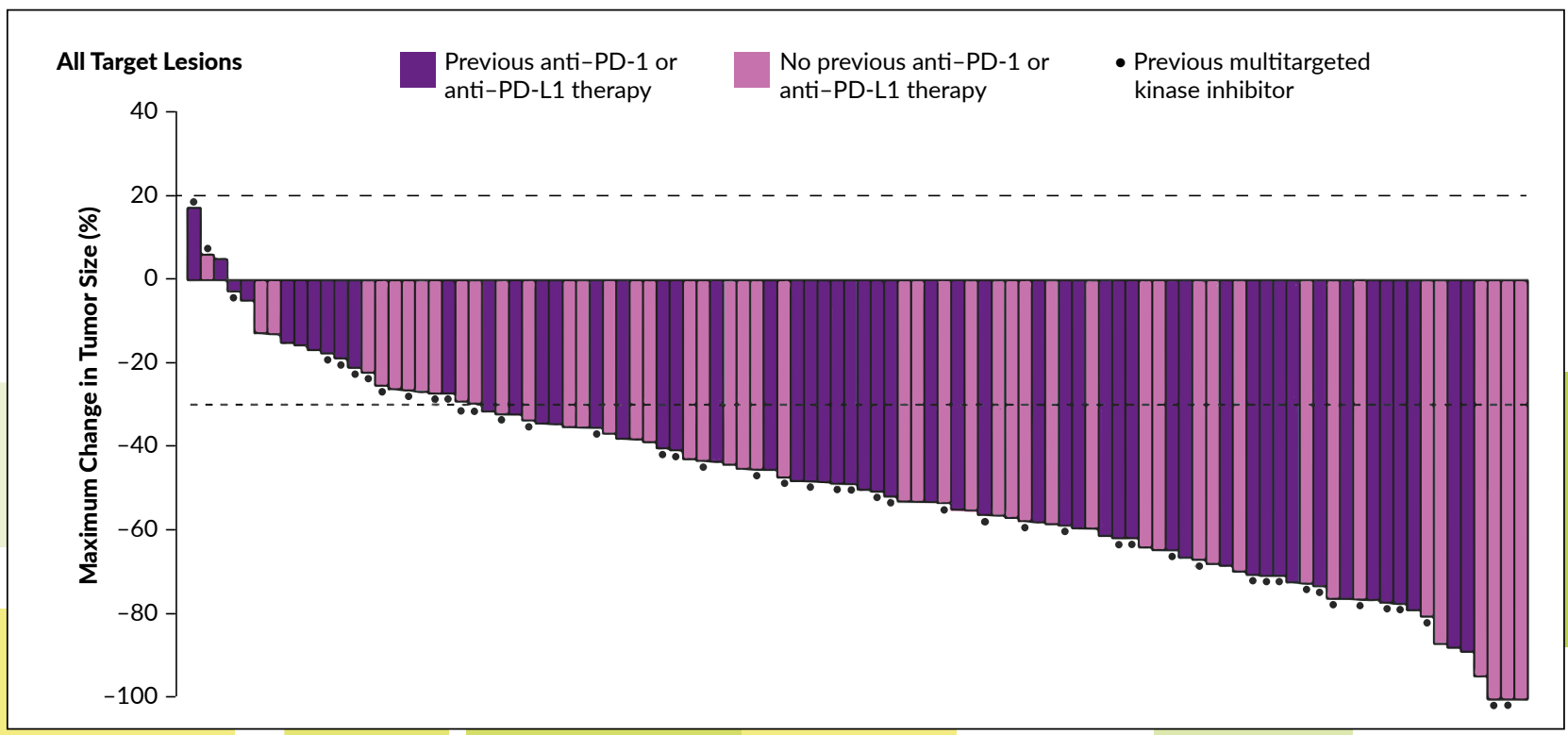

Figure 3. Efficacy data for NSCLC: waterfall plots of the maximum change in tumor size in all target lesions, according to investigator assessment. Adapted from Drilon et al. 2020.3 


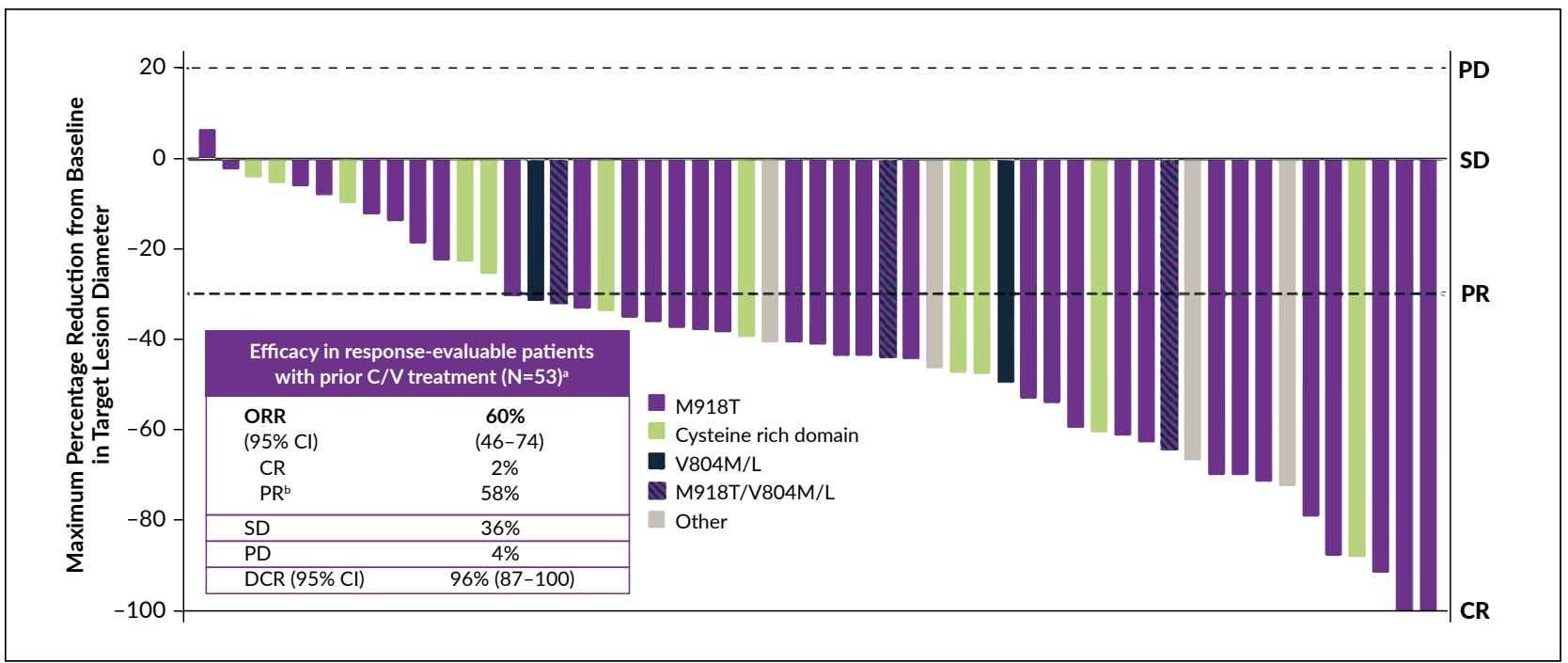

Figure 4. Clinical response to pralsetinib in patients with prior cabozantinib and/or vandetanib treatment. ${ }^{\mathrm{B}} \mathrm{Blinded}$ independent central review of tumor response at data cutoff. Six patients without measurable disease at baseline on central review and 2 patients without a post-baseline tumor response assessment were not response evaluable. ${ }^{b} 1 \mathrm{PR}$ pending confirmation. $\mathrm{C} / \mathrm{V}$, cabozantinib and/or vandetanib; CR, complete response; DCR, disease control rate; ORR, overall response rate; PD, progressive disease; PR, partial response; SD, stable disease. Adapted from Hu et al. 2020.19

The median time to the first onset of TEAEs was within 10 weeks of starting treatment, with the earliest median time to onset for hypersensitivity being 1.7 weeks compared with the latest onset for a rash at 9.3 weeks. ${ }^{17}$ When stratified by the treatment duration ( $<12$ months $[n=466]$ vs $\geq 12$ months $[n=236])$, more patients who received treatment for at least 12 months required dose adjustments due to TEAEs. More specifically, among patients who received selpercatinib $<12$ months versus $\geq 12$ months, $29.6 \%$ and $34.3 \%$, respectively, required dose reduction, $39.5 \%$ and $46.6 \%$, respectively, required dose interruptions, and $6.9 \%$ and $2.1 \%$, respectively, required treatment discontinuation. To ensure manageability of the adverse effects, patients should be carefully monitored, and the dose should be adjusted promptly.

\section{Pralsetinib for RET-altered solid tumors including medullary thyroid cancer}

The current standard of care for treating RET-altered MTC, such as cabozantinib and vandetanib, requires complex dosing regimens and is associated with AEs leading to treatment discontinuation. Pralsetinib (BLU-667) is an orally bioavailable selective inhibitor of wild-type RET and oncogenic RET alterations. ${ }^{8}$ Following the FDA approval of pralsetinib in December 2020, regulatory approval is being sought from the European Commission, with an application to the European Medicines Agency (EMA) and applications for global authorization in the second half of 2021. Preliminary findings from the ongoing phase I/II ARROW study of pralsetinib in patients with thyroid cancer, NSCLC, and other advanced solid tumors have been reported. ${ }^{18,19}$

ARROW is an international, multicenter trial conducted across 84 sites in 11 countries. ${ }^{18,19}$ In this study, eligible patients were those with advanced solid tumors, RET-altered disease per local testing, no other driver mutations, an Eastern Cooperative Oncology Group (ECOG) performance status (PS) of 0 to 1, and those who previously received standard therapy or who were not candidates for standard therapy. ${ }^{19}$ All patients received pralsetinib (400 mg daily) until disease progression or unacceptable toxicity. Four cohorts were included of 67 patients with RET-mutant MTC with prior cabozantinib and/or vandetanib, 42 patients with treatmentnaïve RET-mutant MTC, 10 patients with RET-mutant MTC who had received previous systemic therapy other than cabozantinib and vandetanib, and 319 patients with other RET-altered tumors. The key study endpoints were ORR, DoR, and safety.

At baseline, in the RET-mutant MTC population ( $\mathrm{n}=92)$, the median age was 59 years (range, $19-83$ years) and $68 \%$ of the study population was male. ${ }^{19}$ About $99 \%$ of patients had an ECOG PS of O or 1 and $10 \%$ of patients had a history of CNS/brain metastases. Regarding RET genotype, 61\% of patients had RET M918T-mutant tumors, $29 \%$ had a cysteine-rich domain, 3\% had mutations in RET $V 804 \mathrm{M} / \mathrm{L}$, and the remaining $7 \%$ of patients had other RET alterations. Baseline demographics were comparable between the previously treated and treatment-naïve populations. 
At data cutoff, a high response rate to pralsetinib was observed among 53 previously treated patients. ${ }^{19}$ The ORR was 60\% (95\% Cl: $46-74 \%$ ), with a CR rate of $2 \%$ and a PR rate of $58 \%$

(Figure 4). There were $36 \%$ of patients with stable disease and $4 \%$ with progressive disease, resulting in a disease control rate (DCR) of $96 \%$ (95\% Cl: 87-100\%). Both, the median DoR and the median PFS were not reached. Overall, $75 \%$ of patients remained on treatment at the time of data cutoff, and $94 \%$ of patients with a response continued to receive pralsetinib. In the treatment-naive cohort $(n=42$ ), the ORR was $74 \%$, and the DCR was $100 \%$. Again, both, the median DoR and the median PFS were not reached.

Safety data from ARROW showed that pralsetinib was welltolerated, with a median dose intensity of 92\% (range, 18100\%). ${ }^{19}$ The majority of treatment-related AEs were mild and reversible and most commonly included an increase in serum levels of AST (34\%) and ALT (23\%), anemia (24\%), constipation (23\%), and hypertension (22\%). The most frequent grade 3-4 treatment-related AEs were hypertension (11\%), neutropenia (10\%), anemia (8\%) and a decreased neutrophil count (6\%). Only $4 \%$ of patients discontinued pralsetinib due to treatment-related AEs.
The recent clinical validation of the two highly selective RET inhibitors, selpercatinib and pralsetinib, have shown improved efficacy and safety outcomes. ${ }^{20}$ These new targeted therapies, together with improvements in testing for RET gene alterations in RET-dependent cancers, are set to herald a new era in managing NSCLC and thyroid cancers. The possibility of resistance to these broad-range, next-generation, highly selective RET inhibitors is currently under investigation.

\section{CONCLUSIONS}

- In 2021, ESMO has developed recommendations for the diagnosis of targetable RET rearrangements and mutations. $^{2}$

- Following the results of the phase I/II LIBRETTO-001 trial, selpercatinib has received FDA, followed by Swissmedic approval for RET fusion-positive NSCLC and papillary thyroid cancer and RET mutation-positive medullary thyroid cancer (MTC). 5,6

- Pralsetinib was approved by the FDA for the treatment of patients with lung cancer with RET gene fusion and RET-altered MTC.7.8

\section{Conflict of interest}

A. A. reported receiving personal fees for attending advisory from Bristol-Myers Squibb, AstraZeneca, Roche, Pfizer, Merck Sharp and Dohme, Astellas, Eli Lilly and Boehringer-Ingelheim. He has received fees for speaking bureau for Eli Lilly, AstraZeneca, MSD for work performed outside of the current study. F. S. reported the receipt of personal fees for attending advisory boards from Bristol-Myers Squibb, Roche, Novartis, Pfizer, MSD, Astellas, Ipsen and Incyte.

\section{Authors' contribution}

A. A. has drafted the manuscript; F. S. has critically revised the manuscript. Both authors have approved the final version of the publication.

\section{REFERENCES}

1. Arighi $E$ et al. RET tyrosine kinase signaling in development and cancer. Cytokine Growth Factor Rev. 2005; 16(4-5): 441-67.

2. Belli $C$ et al. ESMO recommendations on the standard methods to detect RET fusions and mutations in daily practice and clinical research. Ann Oncol. 2021; daily practice and
32(3): $337-50$.

3. Drilon A et al. Efficacy of Selpercatinib in RET Fusion-Positive Non-Small-Cell Lung Cancer. N Engl J Med. 2020; 383(9): 813-24.

4. Wirth $\sqcup$ et al. Efficacy of Selpercatinib in RET-Altered Thyroid Cancers. N Engl J Med. 2020; 383(9): 825-35.

5. Food and Drug Administration (FDA) Access Data on Selpercatinib (Retevmo). US Food and Drug Administration. 2020. [Accessed April 2021]. Available ministration. 2020. [Accessed April 2021]. Available docs/label/2020/213246s000lbl.pdf.

6. RETSEVMO ${ }^{\oplus}$ (selpercatinib). Product information. Swissmedic 2021. [Accessed April 2021]. Available from: www.swissmedicinfo.ch.

7. FDA approves pralsetinib for RET-altered thyroid cancers. FDA 2020 [Accessed April 202]. Available from: https://www.accessdata.fda.gov/drugsatfda docs/label/2020/214701s000lbl.pdf.

docs/label/2020/214701s000lbl.pdf.
8. Food and Drug Administration (FDA). Access Data 8. Food and Drug Administration (FDA). Access Data
on Pralsetinib (Gavreto). US Food and Drug Administration. 2020. [Accessed April 2021]. Available from: https://www.accessdata.fda.gov/drugsatfda_docs/label/2020/213721s000lbl.pdf.

9. lams WT, Lovly CM. Stop fRETting the Target: NextGeneration RET Inhibitors Have Arrived. Cancer Discov. 2018: 8(7): 797-9.

10. Salvatore D et al. The importance of the RET gene 10. Salvatore $D$ et al. The importance of the RET gene
in thyroid cancer and therapeutic implications. Nat Rev in thyroid cancer and therapeutic im
Endocrinol. 2021; 17(5): 296-306.

Endocrinol. 2021; 17(5): 296-306.
11. Caprelsa ${ }^{\circledR}$ (vandetanib). Product information. Swissmedic 2021. [Accessed June 2021]. Available from: www.swissmedicinfo.ch.

12. Wells Jr SA et al. Vandetanib in patients with locally advanced or metastatic medullary thyroid cancer: randomized, double-blind phase III trial. J Clin Oncol. randomized, double-blind phase III trial. J Clin Oncol.
2012; 30 : 134-41.

13. Lee $\mathrm{S}-\mathrm{H}$ et al. A phase II study of vandetanib in patients with non-small cell lung cancer harboring RET rearrangement. J Clin Oncol. 2016; 34(15_suppl): 9013.

14. Gainor JF et al. Registrational dataset from the phase I/II ARROW trial of pralsetinib (BLU-667) in patients (pts) with advanced RET fusion+ non-small cell lung cancer (NSCLC). J Clin Oncol. 2020; 38(15 suppl): 9515.

15. Phase $1 / 2$ Study of LOXO-292 in Participants with Advanced Solid Tumors, RET Fusion-Positive Solid Tumors, and Medullary Thyroid Cancer (LIBRETTO-001). ClinicalTrials 2021. [Accessed June 2021]. Available at: https://clinicaltrials.gov/ct2/show/NCT03157128. 16. Subbiah $\vee$, Roszik J. Towards precision oncology in RET-aberrant cancers. Cell Cycle. 2017; 16(9): 813-4. 17. Bauer TM et al. Safety of selpercatinib for RET-altered advanced solid tumors: a post hoc analysis of tered advanced solid tumors: a post hoc analysis of LIBRETTO-001. AACR Annual Meeting 20

April 2021. Poster presentation CT160. hibitor, Pralsetinib (BLU-667), in Patients with Thyroid Cancer, Non-Small Cell Lung Cancer, and Other Advanced Solid Tumors (ARROW). ClinicalTrials 2021. [Accessed June 2021]. Available at: https://clinicaltrials.gov/ct2/show/NCT03037385.

19. Hu $\mathrm{M}$ et al. Results from the registrational phase 1/2 ARROW trial of pralsetinib (BLU-667) in patients 1/2 ARROW trial of pralsetinib (BLU-667) in patients (pts) with advanced RET mutation-positive medullar thyroid cancer (RET+ MTC). 2020 Virtual ESMO Congress; 19- 21 September 2020. Oral presentation 19130

20. Subbiah $\vee$ et al. State-of-the-Art Strategies for Targeting RET-Dependent Cancers. J Clin Oncol. 2020; 38(11): 1209-21.

21. Prazeres $\mathrm{H}$ et al. How to Treat a Signal? Current Basis for RET-Genotype-Oriented Choice of Kinase Inhibitors for the Treatment of Medullary Thy cer. J Thyroid Res. 2011:2011: 678357. 